\title{
Conductometric Hydrogen Gas Sensors Operating at Room Temperature with Irradiation of LED Lights
}

\author{
Takuya Takahashi, Tomiharu Yamaquchi, Kazuhiro Hara \\ Tokyo Denki University, 5 Senjuasahi-cho, Adachi-ku, Tokyo 120-8551 Japan \\ t-yama@mail.dendai.ac.jp
}

\begin{abstract}
:
We present novel conductometric hydrogen gas sensors using $\mathrm{Pt}, \mathrm{Pd}$, and $\mathrm{Ag}$ doped tungsten oxide film operating at room temperature with irradiation of LED lights. Three kinds of LEDs (red, blue and violet) were used in this study. Ammonium paratungstate hydrate solution with addition of various catalysts was spin-coated on an alumina substrate and sintered at $600{ }^{\circ} \mathrm{C}$ for $1 \mathrm{~h}$ in air. When LED lights irradiated the tungsten oxide film, the electron density increased and thus the conductance increased. At the same time, the sensor was sensitive to hydrogen gas at a concentration as low as $100 \mathrm{ppm}$ at room temperature $\left(25^{\circ} \mathrm{C}\right)$. The selectivity to hydrogen gas was satisfactory. The sensor response and recovery times were approximately $190 \mathrm{~s}$ and $270 \mathrm{~s}$, respectively.
\end{abstract}

Key words: hydrogen sensor, room temperature, $\mathrm{WO}_{3}$ film, noble metal catalyst, LED irradiation

\section{Introduction}

A wide variety of hydrogen gas sensors have been investigated including FET-based sensors[1]. Among them conductometric metaloxide sensors have the advantage of a simple structure, small size and low-cost. However, since the semiconductor metal oxide sensors need a heat source, there is a risk of triggering hydrogen explosion.

This paper describes novel hydrogen thick-film gas sensors operating at room temperature with irradiation of LED lights as an energy source promoting the adsorption and reaction on the sensor surface without using a heat source[2].

\section{Sensor Configuration and Fabrication}

A fabricated sensor is shown in Fig. 1. An alumina substrate $(10 \mathrm{~mm} \times 10 \mathrm{~mm} \times 1 \mathrm{~mm})$ was used. The sensor precursor was prepared by mixing $10 \mathrm{~mL}$ of purified water, $1 \mathrm{~g}$ of APT(ammonium paratungstate hydrate), $0.1 \mathrm{~g}$ of PVA(polyvinyl alcohol), $4.4 \mathrm{~g}$ of $\mathrm{Pt}$ nanoparticle colloid (0.2 wt\%), $0.22 \mathrm{~g}$ of $\mathrm{Pd}$ nanoparticle colloid (4 wt \%) and $0.07 \mathrm{~g}$ of $\mathrm{AgNO}_{3}$. It was coated on an alumina substrate by a spin coater at $1000 \mathrm{rpm} 10 \mathrm{~s}$ and successively sintered at $600{ }^{\circ} \mathrm{C}$ for $1 \mathrm{~h}$ in air. Thus $\mathrm{Pt}, \mathrm{Pd}$ and $\mathrm{Ag}$ loaded $\mathrm{WO}_{3}$ thick-film was obtained. The electrode was made by coating Ag paste (Aremco Products, Pyro-Duct 597-A) and treating it at $90{ }^{\circ} \mathrm{C}$ for $2 \mathrm{~h}$.

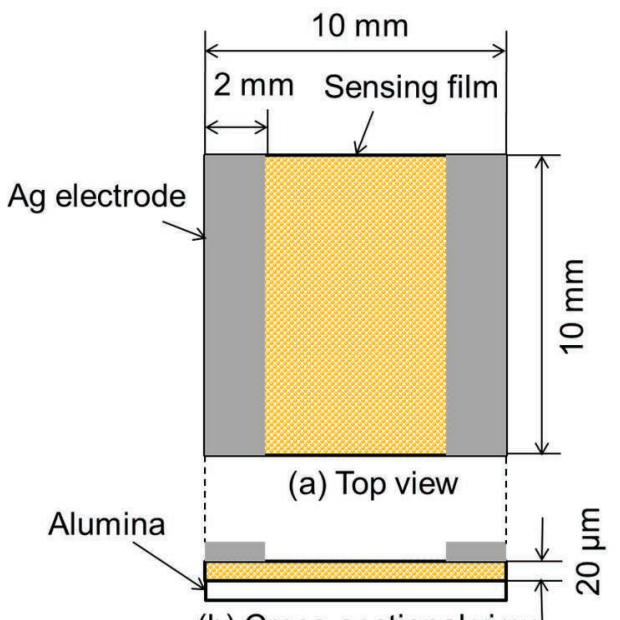

(b) Cross-sectional view

Fig. 1. Schematic view of a fabricated sensor.

The sensor surface was simultaneously irradiated by three kinds of LED lights at an incident angle of about $45^{\circ}$. The characteristics of LEDs (Optosupply) are listed in Table 1.

Tab. 1: Characteristics of the LEDs

\begin{tabular}{|c|c|c|}
\hline Color & $\begin{array}{c}\text { Peak } \\
\text { wavelength }\end{array}$ & $\begin{array}{c}\text { Luminous } \\
\text { intensity }\end{array}$ \\
\hline red & $620 \mathrm{~nm}$ & $100 \mathrm{~cd}$ \\
\hline blue & $470 \mathrm{~nm}$ & $22 \mathrm{~cd}$ \\
\hline violet & $390 \mathrm{~nm}$ & $0.25 \mathrm{~cd}$ \\
\hline
\end{tabular}




\section{Experimental Results}

The sensor resistance was measured using a digital multi-meter (Fluke, 287) under irradiation of LED lights. When LED lights irradiated the tungsten oxide film, the electron density increased and thus the conductance increased. At the same time, the sensor was sensitive to hydrogen gas at room temperature. The dependence of the response on the gas concentration is shown in Fig. 2. Here, the response is defined as the ratio of the conductance in hydrogen-containing air to that in air. The sensor was sensitive at a concentration as low as 100 ppm to hydrogen gas.

Blue and red, violet and red or blue and violet LEDs were irradiated to the sensor for comparison. The sensitivity to hydrogen gas was the best for the case where three kinds of LEDs were simultaneously irradiated.

Three kinds of sensors using Pd and Pt doped, $\mathrm{Pd}$ and $\mathrm{Ag}$ doped or $\mathrm{Pt}$ and $\mathrm{Ag}$ doped $\mathrm{WO}_{3}$ film were also fabricated and tested. None of them was much sensitive to hydrogen gas.

The gas selectivity is shown in Fig. 3. The sensor was sensitive to hydrogen gas among various gases tested. Thus the selectivity to hydrogen gas was satisfactory. The sensor response and recovery times were approximately $190 \mathrm{~s}$ and $270 \mathrm{~s}$, respectively.

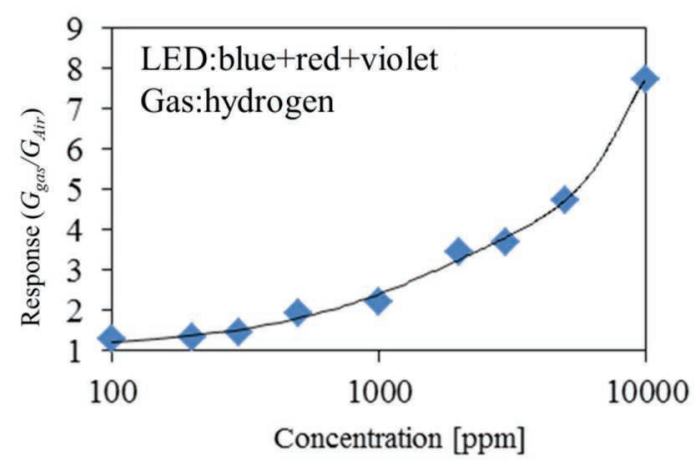

Fig. 2. Dependence of the response on the hydrogen concentration.

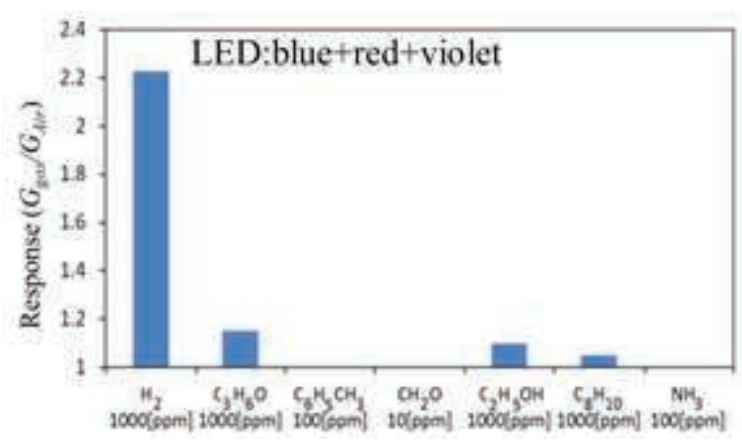

Fig. 3. Gas selectivity of the fabricated sensor.

\section{Discussion}

The fabricated sensor showed high sensitivity to hydrogen at room temperature. A model of the sensing mechanism is illustrated in Fig. 4. Hydrogen molecules tend to dissociate on the $\mathrm{Pd}$ surface with irradiation of blue light and move along the sensor surface by diffusion (known as spill-over effect) to react with superoxide anion $\left(\mathrm{O}_{2}^{-}\right)$which is generated on the $\mathrm{Pt}$ surface with irradiation of violet light and move along the sensor surface by diffusion in similar way. The reaction of $\mathrm{H}$ and $\mathrm{O}_{2}^{-}$is promoted on the Ag surface with irradiation of red light, emitting electron into the sensing film and hence increasing its electrical conductance. Thus three kinds of noble metals along with three kinds of LEDs are effective in enhancing the sensitivity to hydrogen gas at room temperature without a heat source.

The LEDs used were commercially available and inexpensive. Thus the sensor is promising as a leak detector of hydrogen gas which does not cause explosion.

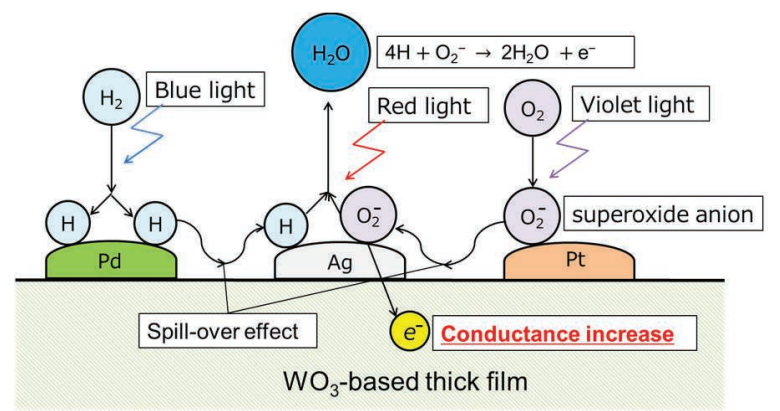

Fig. 4. Model for the sensing mechanism of hydrogen gas at room temperature with irradiation of blue, red and violet LEDs.

\section{References}

[1] K. Tsukada, T. Kiwa, T. Yamaguchi, S. Migitaka, Y. Goto, K. Yokosawa, A study of fast response characteristics for hydrogen sensing with platinum FET sensor, Sensors and Actuators B, 114, 158163 (2006); doi: 10.1016/j.snb.2005.04.026.

[2] O. Tomita, T. Otsubo, M. Higashi, B. Ohtani, R. Abe, Partial Oxidation of Alcohols on Visible-LightResponsive $\mathrm{WO}_{3}$ Photocatalysts Loaded with Palladium Oxide Cocatalyst, ACS Catalysis, 6, 1134-1144 (2016); doi: 10.1012/acscatal.5b01850. 